Urška Valenčič Arh

Oddelek za germanistiko z nederlandtistiko

in s skandinavistiko

Filozofska fakulteta Univerze v Ljubljani

urska.valencic-arh@ff.uni-lj.si
UDK 811.112.2'276.6:355

DOI: $10.4312 /$ vestnik.8.131-149

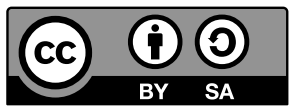

\title{
WAS HABEN FEUERTAUFE, DECKUNG UND STELLUNG GEMEINSAM? - EINIGEN PHRASEMEN AUS DEM SOLDATENALLTAG AUF DER SPUR'
}

Phraseologische Einheiten durchlaufen die sprachliche Evolution so wie alle anderen lexikalischen Einheiten. Phraseme oder einzelne phraseologische Komponenten verändern sich, sterben aus, bekommen neue lexikalische Besetzungen oder erleben Veränderungen in der morphosyntaktischen Struktur. Es gibt auch phraseologische Einheiten, die der Form nach gänzlich oder weitgehend seit ihrer Entstehung bis heute gleich geblieben sind, deren Bedeutung sich aber im heutigen Gebrauch verändert hat (vgl. Burger 2015: 148). Phraseme sind Folgen der festen Verwendung einer Wortverbindung, die in ihrem Entwicklungsstadium aus der konkreten Phase bzw. aus der wörtlichen Bedeutung in die Phase der phraseologischen Bedeutung übergegangen war. In einer Sprachgemeinschaft nimmt der Gebrauch der Wortverbindung in ihrer phraseologischen Bedeutung immer mehr zu, während der Gebrauch derselben Wortverbindung in der literalen, d. h. wörtlichen Bedeutung immer mehr in den Hintergrund tritt. Letztendlich gewinnt die übertragene, phraseologische Bedeutung die Oberhand. ${ }^{2}$ In diesem Prozess verlieren Phraseme auf der semantischen, lexikalischen oder grammatikalischen Ebene ihre ursprüngliche Rolle. In unserer Untersuchung wollen wir der Historizität einiger Wortverbindungen nachgehen, die im Soldatenalltag zur Benennung oder Bezeichnung bestimmter Sachverhalte

\footnotetext{
1 Für den vorliegenden Beitrag konnte ich im Rahmen meines Studienaufenthaltes in der Bibliothek des Institutes für Slawistik an der Karl-Franzens-Universität Graz recherchieren und wertvolle Informationen sammeln. Ich möchte mich dafür ganz herzlich bei Frau Mag. Kasilda Bedenk und Herrn Dr. Heinrich Pfandl für die zuverlässige Betreuung und bei der Institutsleitung Frau Univ.-Prof. Dr. Renate Hansen-Kokoruš für die freundliche Unterstützung bedanken.

2 Ein Beispiel dafür, wie einzelne Lexeme langsam aus dem alltäglichen Sprachgebrauch verschwinden, konnte bei der Arbeit mit meinen Deutschstudierenden in Ljubljana festgestellt werden. Es ging um das Phrasem vom Regen in die Traufe kommen in der Bedeutung 'aus einer unangenehmen Lage in eine noch unangenehmere geraten'. Nach einer kurzen Umfrage unter den Deutschstudierenden stellte sich heraus, dass die jungen Leute (23 Personen im Alter von 21 Jahren) die phraseologische Bedeutung nicht erschließen konnten, obwohl es in der slowenischen Sprache ein adäquates Phrasem aus dem gleichen Bildbereich gibt: $z$ dežja pod kap. Sie haben das Wort Traufe (slow. kap) weder im Deutschen noch im Slowenischen verstanden.
} 
oder Tätigkeiten verwendet wurden, deren wörtliche Bedeutung aber im Laufe der Zeit in Vergessenheit geraten ist. In einem authentischen Text aus der Zeit des 1. Weltkrieges sind wir auf Ausdrücke in ihrer wörtlichen Bedeutung gestoßen, die uns in ihrer textuellen Umgebung verständlich vorkamen, obwohl wir sie aus der gegenwärtigen Verwendung nur in ihrer phraseologischen Lesart kennen.

In der vorliegenden Untersuchung fragen wir nach der historischen Bedeutung des jeweils untersuchten Phrasems, das heißt, wir wollen die ursprüngliche semantische Basis, die Burger (2015: 193) als historische „Motivationsbasis“ bezeichnet, an ihrem historischen Platz erforschen. Es interessiert uns sowohl die diachrone als auch die synchrone Perspektive der Betrachtung, so dass wir diese Ausdrücke aus einem Kriegstagebuch und Belege aus Texten der Gegenwart einem Vergleich unterziehen. Ausschlaggebend sind dabei Fragen der Veränderung in der lexikalischen Besetzung und Semantik. Beim methodischen Vorgehen werden auch die relevanten Parameter (Autor, historischer Hintergrund) in der Bestimmung der Kommunikationssituation für die Textsorte Tagebuch beschrieben.

\section{DIE SEMANTISCHE BASIS DER PHRASEME}

In der Phraseologieforschung werden Phraseme als vorgeprägte bzw. lexikalisierte und somit reproduzierbare sprachliche Zeichen definiert, die syntaktisch und semantisch (relativ) stabil sind. Ihre Bedeutung ist ganzheitlich und wird nicht aus der primären, wörtlichen Bedeutung der einzelnen Komponenten und aus deren syntaktischen Beziehungen zueinander erschlossen. Dieses Merkmal wird Idiomatizität genannt. Idiomatizität tritt auf, wenn eine Verbindung von zwei oder mehreren Wörtern syntaktisch und semantisch nicht voll erklärbar ist und die Summe der Bedeutungen der einzelnen Komponenten in den meisten Fällen keinen Sinn ergibt (vgl. Burger et al. 1982: 1). Man unterscheidet den phraseologischen Kernbereich, wenn die Merkmale Polylexikalität, Festigkeit und Idiomatizität vorhanden sind, und den peripheren phraseologischen Bereich, in dem sich phraseologische Einheiten befinden, die keine Idiomatizität aufweisen.

In der Fachliteratur wird das Merkmal der Idiomatizität mit dem Begriff der Motiviertheit in Verbindung gebracht. Beide Phänomene stellen semiotische Gegenpole zueinander dar: „Je idiomatischer ein Phraseologismus, desto weniger motiviert ist seine phraseologische Bedeutung. Idiomatizität und Motiviertheit sind umgekehrt proportional.“ (Stöckl 2004: 165). Laut Burger (2015: 67) sind Phraseme synchron verstehbar, wenn sie motiviert sind. Hinsichtlich der semantischen Perspektive geht es bei der Motiviertheit um

„den Anteil, den jeweils die wörtliche Bedeutung der ganzen Wortverbindung oder einer Komponente am Zustandekommen und damit der „Verstehbarkeit“ der phraseologischen Bedeutung hat.“ (ebd.) 
Als nicht motiviert und starkt idiomatisch gelten z. B. Phraseme aus dem Militärbereich in Harnisch sein ('zornig sein') ${ }^{3}$, jmdn. in Harnisch bringen ('jmdn. so reizen, dass er zornig und wütend wird') und in Harnisch geraten/kommen ('im Verlauf eines Ereignisses wütend, zornig werden'), weil die semantischen Basen fehlen und der Sprecher unter der synchronen Perspektive keinen Zusammenhang herstellen kann, wenn ihm die Bildlichkeit nicht „einleuchtet“. Im Bewusstsein der Sprecher oder als konkreter Gegenstand existiert Harnisch wohl nicht mehr. Um zu verstehen, dass mit dem Harnisch die Ritterrüstung gemeint ist und dass man diese Rüstung trägt, wenn man zum Kampf bereit ist, weil man verärgert oder durch Ärger und Zorn erregt ist, muss dem Sprecher der gesamte Hintergrund der in den einzelnen Komponenten ,versteckten Geschichte bekannt sein. ${ }^{4}$ Als motivierte Phraseme werden z. B. Phraseme wie aus der Pistole geschossen ('ohne langes Überlegen, ohne Zögern') und jemandem die Pistole auf die Brust setzen ('jmdn. ultimativ zu einer Entscheidung zwingen') betrachtet bzw. eingestuft. Bei diesen kann der Sprecher die Bedeutung der festen Wortverbindung aufgrund der einzelnen figurativen Szenen und der Bildlichkeit verstehen, weil die einzelnen Lexeme in ihrer ursprünglichen Bedeutung im allgemeinen Sprachgebrauch heute noch verwendet werden. Im Hinblick auf die semantische Perspektive trägt die wörtliche Bedeutung der ganzen Wortverbindung oder eines Teils bzw. einer Komponente zum Verstehen der ganzen Wortverbindung bei. In diesem Fall wird die phraseologische Bedeutung synchron erschlossen. Burger (2015: 67) spricht von der semantischen Basis des Phrasems:

„Wir bezeichnen diejenigen Wörter oder Wortkomplexe, die in ihrer freien Bedeutung am Zustandekommen der phraseologischen Bedeutung beteiligt sind, als die semantische Basis des Phrasems.“

Als motiviert und schwach idiomatisch gelten demnach diejenigen Phraseme, die eine semantische Basis haben. Zwischen den beiden Polen befindet sich auch noch die Gruppe der teil-motivierten bzw. teil-idiomatischen Phraseme. Bei diesen ist nur ein Teil der festen Wortverbindung für den Sprecher verstehbar. Die partielle Motiviertheit zeigt sich oft in Phrasemen, in denen (nur) einzelne Komponenten heute nicht mehr in ihrer ursprünglichen Bedeutung verwendet werden, jedoch kann man aus den übrigen Teilen des Phrasems intuitiv die Bedeutung erschließen, wie z. B. bei komparativen Phrasemen wie voll/betrunken/blau wie eine Haubitze/Strandhaubitzes sein ('völlig betrunken sein'). In der Phraseologie tauchen auch Begriffe der Demotivation und Remotivation auf. Mit der Demotivation ist das Phänomen des lexikalischen historischen Wandels gemeint.

3 Der Harnisch ist eine Ritterrüstung. Die Bedeutungserklärungen stammen aus DUDEN 11 und Duden Wörterbuch (duden.online).

4 Für diese Beobachtung verwendet man in der Phraseologieforschung auch die Begriffe ,opak” und „transparent” oder „durchsichtig”. Als opak oder undurchsichtig gelten Phraseme mit einem hohen Grad an Idiomatizität und als transparent oder durchsichtig die Phraseme mit einem niedrigen Grad an Idiomatizität.

5 Die Haubitze: Geschütz mittleren oder schweren Kalibers mit kurzem Rohr (Duden) 
Wortverbindungen werden demotiviert, weil sie „,in eine Asynchronität von Bedeutung und soziokulturellem Kontext geraten" (Stöckl 2004: 166). Zur Zeit ihrer Entstehung und Bildung sind fast alle Phraseme motiviert, jedoch verlieren einige durch den Wandel der Lebensumwelt und in der Evolution einzelner Komponenten ihre Motivation (vgl. ebd.). Phraseologische Einheiten besitzen nicht nur ihre denotative Bedeutung, sondern sie drücken als Träger subjektiver Expressivität auch gleichzeitig Konnotationen aus. Während die Demotivation historisch bedingt ist, ist die Remotivation eine vom Sprecher oder Schreiber bewusst durchgeführte semantische Aktivierung der möglichen wörtlichen Lesarten und stellt ein textlinguistisches Phänomen dar, unter anderem auch im Sinne des modifizierten Gebrauchs (vgl. Burger 2015: 68f.).

Zwischen der phraseologischen und der freien bzw. wörtlichen Bedeutung kann (meistens) ein Zusammenhang bestehen, was fast immer mit einem Bild oder einer Vorstellung zusammenhängt, die uns hilft, die phraseologische Bedeutung zu erkennen und zu verstehen. Diese Bilder werden aus den unterschiedlichsten Bereichen des menschlichen Lebens in die Kommunikation übernommen, einige davon auch aus dem Militärbereich. In der weiteren Untersuchung wollen wir Wortverbindungen nachgehen, die aus dem Soldatenalltag stammen und im Laufe der Zeit zum festen Bestandteil des allgemeinen Wortschatzes wurden.

\section{DAS TAGEBUCH ALS AUTHENTISCHES ZEUGNIS UND HISTORISCHE QUELLE}

Die literaturwissenschaftliche Forschung zählt das Tagebuch zu den Formen eigener Lebenserzählung (Dusini 2006), in der das Erlebte festgehalten und „von einer Reflexion auf das Ich“(Wuthenow 1990: 1) geprägt wird. Als Selbstzeugnis wird das Tagebuch den autobiographischen Gattungen, wie Autobiographie, Brief und Memoirenliteratur zugeordnet (vgl. Dusini 2005, Wuthenow 1990). In der Forschung wird eine weitere Differenzierung vorgenommen: Es wird zwischen zwei Grundtypen von Tagebüchern unterschieden: dem authentischen und dem literarischen Tagebuch. Die Unterscheidungsmerkmale betreffen die Absicht des Autors, seine Aufzeichnungen zu veröffentlichen oder nicht und die „Überformung des schriftlich Fixierten“ als maßgebliches Element der Literatizität (vgl. Hardtwig 2005: 150). Die Diskussion über das Private oder nicht Private bzw. Dienstliche hängt von den Rezeptionsbedingungen, denen das Tagebuch unterliegt, und der Verfügbarkeit der Schrift ab. Private Tagebücher dienen der selbstreflexiven Auseinandersetzung in einer Intimsphäre, die eine Interaktion im Sinne einer kommunikativen Handlung mit konkreten Rezipienten ausschließt. Zu den nicht-privaten Selbstzeugnissen gehören auch Kriegstagebücher, die neben Feldpostkarten, -briefen oder Soldatenzeitungen als historische Quellenbestände bezeichnet werden (Wisthaler 2011: 7). Diese Selbstzeugnisse werden wiederum unterschiedlich bewertet, je nachdem, ob es sich um 
echte oder retrospektive Kriegstagebücher handelt. Bei den Retrospektiven können die Rückblicke nur schwer rekonstruiert werden, weil Notizen Jahre später inhaltlich und formal aufgrund des zeitlichen Abstands verändert werden können. Dagegen liegen die echten Kriegstagebücher in der Originalform vor und zeichnen sich durch ihre Nähe zum Erlebten und die überlieferten Emotionen aus.

Beim untersuchten Tagebuch Franz Dobniks handelt es sich um ein authentisches privates Kriegstagebuch, das die Erlebnisse vor Ort dokumentiert. Einige Notizen, die vor allem an seine Familie gerichtet waren, weisen darauf hin, dass sich der Autor darüber bewusst war, dass sein dokumentiertes Selbstzeugnis von den Familienangehörigen und den Vorgesetzten gelesen werden wird. ${ }^{6}$ Wir betrachten den untersuchten Tagebuchtext als ein teils privates, teils dienstlich spontan erzeugtes Text-Ganzes mit einzelnen Vertextungsstrategien, wie z.B. Beschreiben, Erzählen, Bewerten, Argumentieren usw., die in der Auswahl der einzelnen Themen, des linguistischen Beschreibungsinventars sowie der sprachlichen Mittel zum Ausdruck kommen.

Franz Dobnik wurde am 8. August 1871 als Sohn einer Bauernfamilie in dem kleinen Ort Letuš in Savinjska dolina in Slowenien geboren. Er besuchte die Landwehrkadettenschule in Wien. ${ }^{7}$ Am 1. Oktober 1901 trat er dem neuformierten Landwehr-InfanterieRegiment (LIR) 27 in Ljubljana/Laibach bei, das zu den K.K. Gebirgstruppen gehörte. ${ }^{8}$ Bedingt durch die Struktur des Vielvölkerstaates und demzufolge der bunten ethnischen Zusammensetzung in fast allen Regimenten, bestand das LIR 27 zum größten Teil (zu 86\%) aus Slowenen. ${ }^{9}$ Franz Dobnik wurde im I. Weltkrieg als Hauptmann nach Galizien an die Ostfront geschickt. Nach offiziellen Quellen des österreichischen Staatsarchivs ist er am 15. Dezember 1914 bei Skolyzin in Polen gefallen, nach Angaben seiner Verwandten und des Bild- und Tonarchivs am Landesmuseum Joanneum in Graz aber am 16. Dezember 1914 auf dem Schlachtfeld von Zmigrod bei Jasło in Galizien. Seine Tagebuchaufzeichnungen hatte Franz Dobnik zwischen dem 15. August 1914 und dem 15. Dezember 1914 im Feld in Galizien in ein kleines Notizbuch notiert.

6 „Bitte das Tagebuch, falls ich falle, meiner Frau (Adresse: Elsy Dobnik, Görz, via Dogana 22) zu übergeben.“ (Dobniks Tagebuch, erste Seite); „Elsy siedelt vielleicht gerade und ist in großer Angst wahrscheinlich. Ich möchte ihr gern sagen, daß ich noch am Leben bin, daß ich schon zweimal im heftigen Feuer war, doch sagen möchte ich ihr nicht, daß ich heute das dritte Mal vielleicht hineinkomme und mit sehr geringer Aussicht auf einen Erfolg. Vom Tagebuch bekommt sie ja später doch Kenntnis." (Dobniks Tagebuch, 2.9.1914)

7 Österreichisches Staatsarchiv URL: http://www.archivinformationssystem.at/detail.aspx?ID=88128

8 Ab dem 11. April 1917 hieß es k.k. Gebirgs-Schützenregiment Nr. 2.

9 Bis dahin befanden sich in Laibach/Ljubljana zwei Bataillone des Klagenfurter Landwehr-Infanterie-Regiments (LIR). (Vgl. Stergar 1999:75) 


\section{$5 \quad$ ANALYSE DER SEMANTISCHEN BASIS}

Um die Fragen nach den Motivations- bzw. semantischen Basen der phraseologischen Einheiten beantworten und ihre eventuellen Veränderungen in der Struktur und Bedeutung unter diachronem Aspekt beschreiben zu können, werden phraseologische Einheiten nach folgenden methodologischen Schritten demonstriert und kommentiert: lexikalisierte Nennform(en) des Phrasems, verschiedene Lesarten laut Duden-Wörterbuch, Belege aus dem Kriegstagebuch Dobniks und Belege aus dem aktuellen Gebrauch. Der Ausgangspunkt ist ein Inventar von phraseologischen Einheiten mit den Komponenten Feuertaufe, Deckung und Stellung aus dem Militärbereich, die dem Tagebuch entnommen und in deutschen Wörterbüchern (Duden - Das große Wörterbuch der deutschen Sprache in 10 Bänden, DUDEN 11, Röhrich, Neumann, Grimm) nachgeprüft werden. Das empirische Material setzt sich aus den Tagebuch-Originalbelegen und den Belegen aus den DWDSKorpora (Referenzkorpora und Zeitungskorpora) ${ }^{10}$ zusammen. In Zweifelsfällen werden zusätzlich auch andere Quellen in Betracht gezogen.

$$
\begin{aligned}
& \text { die Feuertaufe bestehen (Neumann), (Duden) } \\
& \text { die Feuertaufe empfangen (Grimm), (Dobnik) } \\
& \text { die Feuertaufe erhalten (Röhrich) }
\end{aligned}
$$

LESART 1: 'erste Bewährungsprobe‘

Beleg 1 Die erste männliche Lottofee hat ihre Feuertaufe bestanden.

(Die Zeit, 3.7.2013)

Beleg 2 Enterprise: Im August 1977 hatte das neue US-Raumfahrt-Programm seine Feuertaufe.

(Der Tagesspiegel, 3.2.2003)

LESART 2: 'erste Teilnahme eines Soldaten an einem Gefecht/
zum ersten Male dem feindlichen Feuer ausgesetzt sein"

Beleg 3 Das Pfeifen der Geschosse machte mir nichts, ich fühlte mich so ziemlich sicher. Aber der Anblick der toten Soldaten und Pferde, sowie das

\footnotetext{
10 In den Referenzkorpora sind vertreten: das Deutsche Textarchiv, das deutschsprachige Texte aus dem Zeitraum von ca. 1600 bis 1900 bereitstellt, das DWDS-Kernkorpus mit Texten des 20. Jahrhunderts und das DWDSKernkorpus 21 mit Texten aus den Jahren 2000 bis 2006 (insgesamt: 94.370 Dokumente). Unter Zeitungskorpora werden Artikel aus drei Zeitungen in digitaler Form zur Verfügung gestellt: aus der Berliner Zeitung aus der Zeit vom Januar 1994 bis Dezember 2005, aus dem Tagesspiegel zwischen 1996 und Juni 2005 und aus der ZEIT von 1946 bis heute (insgesamt 2.302.009 Dokumente) (Vgl. http://www.dwds.de/r)
} 
Röcheln sterbender Menschen und Tiere und das Jammern Verwundeter war mir fürchterlich. An das werde ich mich kaum gewöhnen können. Ich habe nun die Feuertaufe empfangen.

(Tagebuch Dobniks, 26.8.1914)

LESART 3: 'zum ersten Mal erteilen', 'reinigen, neue Kraft erhalten' (hist.)

Beleg 4 Tauff-Sermon, Von der Christen Geist- und Feuer-Tauffe, abgeleget Vor dem Fürstlichen Wochen-Bette auf der Residentz zu Friedenstein, Als die Neugebohrne Durchl. Sachsen-Gothaische Princeßin, Princeße Sophia Hertzogin zu Sachsen, Jülich, Cleve und Berg ... Zu dem Heiligen Gnaden-Bunde mit Gott Den 24. Aug. An. 1712. gefodert wurde. : Auf Hoch-Fürstlichen gnädigsten Befehl anjetzo dem Druck übergeben / Von D. Johann Heinrich Feustking, Fürstl. Sachsen-Gothaischen OberHof-Prediger, Beicht-Vater, Ober-Consistorial- und Kirchen-Rath [...].

(Taufpredigt auf die Sachsen-Gothaische Prinzessin Sophia, Tochter von Friedrich II., 24.08.1712) ${ }^{11}$

Für die metaphorische Deutung der ,Feuertaufe` bestehen mehrere Erklärungen. Die unterschiedlichen Interpretationen der Motivationsbasis sind auf semantische Unterschiede in der Motivation der Lexeme Feuer und Taufe sowie die Frage, was diese beiden Lexeme eigentlich verbindet, zurückzuführen. Gemeint sind entweder Taufe(n) mit Feuer (= reinigen, neue Kraft erhalten) oder Feuertaufe (= Bewährungsprobe). ${ }^{12}$

Die Verbindung der Komponenten Taufe und Feuer geht ursprünglich auf die Worte von Johannes dem Täufer zurück, der in der Bibelstelle Matth. 3,11 (vgl. Lukas 3,16) die Ankunft von Jesus verkündet: „Ich taufe euch mit Wasser zur Buße; der aber nach mir kommt, ist stärker denn ich, dem ich nicht genugsam bin, seine Schuhe zu tragen; der wird euch mit dem Heiligen Geist und mit Feuer taufen." (vgl. Kluge, 1989:212; Piirainen 2012:222) Dem Feuer wird hier Reinigungskraft zugeschrieben. Im Deutschen Wörterbuch von Jacob und Wilhelm Grimm (1854-1961) findet man jmdm. die Feuertaufe erteilen im Sinne von 'etwas wird jmdm. zum ersten Mal erteilt' (ebd. 1862: 1606). Die Lesart 3, die hier als historisch gekennzeichnet wird, wird im Beleg 4 bestätigt. Es geht um die Taufpredigt für die Tochter von Friedrich II. aus dem Jahr 1712. Wie aus dem von

11 Quelle: Digitalisierung von Drucken des 18. Jahrhunderts Halle, Saale : Universitäts- und Landesbibliothek Sachsen-Anhalt, 2010 (http://digitale.bibliothek.uni-halle.de/id/1656695)

12 Neumann (2016: 54) erwähnt in diesem Zusammenhang auch die Feuerprobe, mit der man im Mittelalter die Schuld oder Unschuld eines Beschuldigten feststellen konnte. Ein Beschuldigter wurde ,probeweise dem Feuer übereignet“ (ebd.) und wenn er unschuldig war, wurde das arme Opfer von Gott geschützt. Röhrich (1995: 44) sieht dagegen den Ursprung der Wortverbindung die Feuerprobe bestehen in der Prüfung von Edelmetallen, besonders von Gold, durch Feuer. 
Röhrich (1995: 441) angeführten Zitat von H. J. Campe aus dem Jahre 1808 hervorgeht, bedeutete der Ausdruck Feuertaufe auch 'übernatürliche geistliche Gaben erhalten'.

Als der englische Ausdruck baptism of fire aus dem französischen baptême du feu in der Bedeutung 'erste Schlacht des Soldaten' entlehnt wurde, kam es zur Umdeutung der semantischen Basis der Wortverbindung (vgl. Piirainen 2012: 222). Dem Feuer wurde die Bedeutung von 'Schlacht, Kampf' zugeschrieben und dem Lexem Taufe die Bedeutung der 'ersten Erfahrung'. Auch ein Offizier wurde in den Ritterstand erhoben, wenn er tatsächlich ein Feuergefecht mit Feuerwaffen bestand. Die Wortverbindung jmd. empfängt die Feuertaufe wurde auch schon in Grimms Wörterbuch im Zusammenhang mit dem Kriegsgeschehen paraphrasiert 'vom Krieger, der zuerst im Feuer steht' (Grimm 1862: Bd. 3, Sp. 1606). Im Beleg 3 schildert Dobnik in seinem Tagebuch das traumatische Erlebnis seiner Feuertaufe an der Ostfront. Seine Beschreibung zeugt von starken Emotionen, einer sehr niedergeschlagenen Stimmung und wirkt bedrückend, fast frustrierend auf den Leser. In der Beschreibung der Situation, in der er zum ersten Male dem feindlichen Feuer ausgesetzt war, das hier in der literalen Bedeutung und gleichzeitig als semantische Basis aufgefasst werden kann, verwendet er das Verb empfangen, das hier neutral wirkt. Nach dem DWDSWortprofil wird das Verb empfangen im aktuellen Gebrauch erst an der 5. Stelle verzeichnet. Die häufigsten Verben, die im lexikalischen Umfeld des Wortes Feuertaufe vorkommen, sind: bestehen, überstehen, erleben, ausstehen, empfangen, erfahren, feiern, erhalten. Am häufigsten werden positiv konnotierte Verben gebraucht, z.B. bestehen im Beleg 1. Die Wahl der Verb-Komponente scheint sehr variabel zu sein. Der Grund dafür liegt wahrscheinlich in der starken semantischen und motivierten Präsenz des Lexems Feuertaufe. Heute hat sich der Ausdruck Feuertaufe als phraseologische Komponente in der Bedeutung 'erste Bewährungsprobe, eine Art Initiation' stabilisiert. Die zweite figurative Bedeutung bleibt im Sinne 'im Krieg die erste Schlacht erleben, zum ersten Mal beschossen werden' (vgl. Röhrich 1995: 441) lexikalisiert.

$$
\text { in Deckung + Verb }
$$

Im Duden-Wörterbuch werden dem Lexem Deckung sieben Bedeutungserklärungen zugewiesen: deckende Schicht, schützen, Befriedigung der erforderlichen Mittel, Absicherung im kaufmännischen Sinne, das Sichdecken, das Decken oder sich Verteidigen im Sport und das Gedecktwerden. Im Bereich des Militärs wird beim Lemma Deckung weiterhin zwischen drei Bedeutungen unterschieden: 'das Schützen, Sichern und Abschirmen', 'Schutz gegen Sicht oder Beschuss' oder 'Verheimlichung einer ungesetzlichen Handlung'. Im Zusammenhang mit Schutz oder Verteidigung bezeichnet der Ausdruck in Deckung eine Position, die vor Gefahr schützt. Wenn man sich angegriffen fühlt, dann geht man in Deckung und bringt sich in Sicherheit. Diese konkret motivierte Lesart, sich vor Gefahr schützen' kommt in Dobniks Eintragungen in der Wortverbindung in Deckung mit den Verben sich verkriechen, bleiben und kommen an drei Textstellen vor. 
Beleg 5 Ca. $1 \mathrm{~h} 30$ erfolgt ein schwacher Feuerüberfall, d.h. es ist möglich, daß Patrouillen auf unsere befestigten Linien stießen, nicht unwahrscheinlich ist es jedoch, daß auf die eigenen Patrouillen ein mörderisches Feuer eröffnet wurde. Das brachte große Beunruhigung hervor. Die Mannschaft verlief sich im Wald oder verkroch sich in die schützende Deckung.

(Tagebuch Dobniks, 29.8.1914)

Beleg 6 Morgens geht wieder alles an die natürliche Deckung heran. Im Laufe des Vormittags verschieben wir uns etwas nach rechts und vorwärts und graben uns ein. [...] Das III. Baon ist ebenfalls dabei, während II. noch immer geschützt in Deckung bleibt.

(Tagebuch Dobniks, 17.10.1914)

Beleg 7 Bei hellichten Tag kommt erst ... Kommandant Oberleutnant Straschek und Masch. Gew. Abt. Kommdt. Oberleutnant Paulus in die Deckung. Das Baon ist zurückgeblieben, gedeckt im Walde hinter Budy.

(Tagebuch Dobniks, 26.10.1914)

Im Kriegstagebuch befinden sich in naher Umgebung zur Betonung der gefährlichen Lage weitere Lexeme, die die Notwendigkeit eine Gefährdung abzuhalten, intensivieren: schützende Deckung, geschützt in Deckung. Der konkrete Gegenstand, der einem Schutz bieten kann, wird weder in Dobniks Tagebuch noch in den gegenwärtigen Belegen konkret verbalisiert. Genauso findet sich im Tagebuch-Korpus keine andere Motivation als 'den Schutz suchend bzw. bietend".

Im DWDS-Korpus gehen die meisten Belege mit der Wortverbindung in Deckung + Verb auf die Motivation 'Schutz suchen' zurück. Unter dem strukturellen Blickpunkt wird in Dobniks Tagebuch keine Verbindung mit dem Verb gehen gefunden, während im DWDSKorpus das Verb gehen am häufigsten als Komponente eingesetzt wird, gefolgt von bringen, bleiben, liegen, sein, ziehen und halten, wie aus Graph 1 ersichtlich ist.

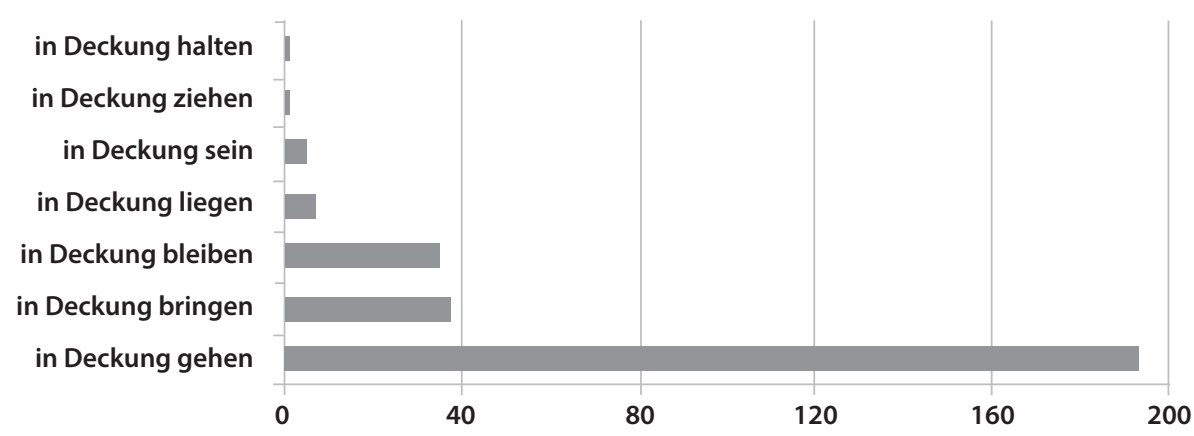

Graph 1: Die feste Struktur in Deckung mit unterschiedlichen Verb-Komponenten 
Im weiteren Verlauf der Analyse zeigten sich unterschiedliche Lesarten der einzelnen Wortverbindungen, je nachdem welches Verb die freie Verbstelle besetzte. Aus diesem Grund werden sie im Weiteren auch einzeln dargestellt.

(a) in Deckung gehen (DUDEN 11), (Duden), (Neumann)

LESART 1: 'sich verstecken', 'Schutz gegen Sicht oder Beschuss suchen'

Beleg 8 Vielleicht würde ich, ein Tourist, dem offenbar das starke Sonnenlicht nicht gut bekommt, in Deckung gehen, und im Schatten unter den Arkaden bliebe mir nichts anderes, als genau die Stelle zu erkennen, wo unser Großvater seinen ersten Toten zu Gesicht bekommen hat.

(Beyer, Marcel: Spione, Köln: DuMont 2000: 184)

LESART 2: 'sich vor etwas drücken"

Beleg 9 Wer also ist dieser Andreas Heeschen, der über seinen Lebenslauf beharrlich schweigt und in Deckung geht, sobald ihn jemand danach fragt?

(Die Zeit, 22.08.2013)

Im ursprünglichen Sinne war mit der Wortverbindung in Deckung gehen gemeint, dass man bei Beschuss durch feindliche Waffen sein Leben rettet und Schutz sucht. Den Befehl „Volle Deckung!“ haben alle Soldaten zu allen Zeiten verstanden. Neumann (2016: 50) fügt die Bedeutung im umgangssprachlichen Gebrauch ,sich vor etwas drücken“ hinzu, die dann verwendet wurde, wenn im Soldatenalltag Freiwillige für unangenehme Aufgaben gesucht wurden. Die beiden oben genannten Lesarten lassen sich im DWDSKorpus nur schwer voneinander trennen, so dass erst die kontextuelle Umgebung den Hinweis auf die eine oder andere Bedeutung liefert. Von den 192 Belegen im DWDSKorpus werden 176 (92\%) der ersten Lesart zugeordnet, und nur ein kleiner Anteil von 8 Prozent wird gegenwärtig in der Bedeutung 'sich vor unangenehmen Aufgaben drücken" eingesetzt (vgl. Graph 2).

in Deckung gehen (DWDS-Korpus)

- 'sich verstecken', 'Schutz gegen Sicht oder Beschuss suchen'

'sich vor etwas drücken'

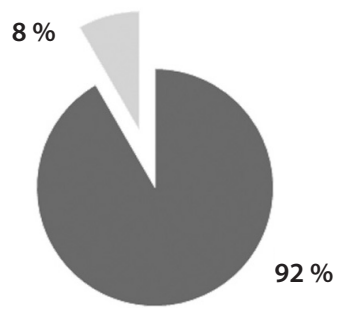

Graph 2: Der Anteil der Lesarten von in Deckung gehen im DWDS-Korpus 
LESART 1: 'sich verstecken', 'Schutz gegen Sicht oder Beschuss suchen' (DUDEN 11)

Beleg 10 Dort wacht er schwer verletzt und ohne Erinnerung in einem Krankenhaus auf - und muss sich gleich vor einer Auftragskillerin in Deckung bringen.

(Die Zeit, 14.05.2013)

LESART 2: 'sich decken, gleich sein, unterschiedliche Standpunkte in Einklang/ zur Deckung bringen“

Beleg 11 Die Höhe der Entgelte verhandeln Lufthansa und die Pilotenvereinigung Cockpit, und wie immer wird am Ende ein Kompromiss stehen, der die Möglichkeiten des Unternehmens mit den Bedürfnissen der Piloten in Deckung bringt.

(Der Tagesspiegel, 28.03.2001)

Im Hinblick auf den aktuellen Gebrauch, unter synchronem Aspekt also, verfolgt ein Sprecher mit der Wahl der praseologischen Einheit in Deckung bringen den Zweck, entweder etwas zu verstecken oder unterschiedliche Standpunkte einander so anzupassen, dass sie zufriedenstellend und überzeugend zusammen- und nicht auseinanderfallen. Man kann sich unter den einzelnen Komponenten leicht bildhaft vorstellen, wie zwei Sachen zusammen gebracht werden. Auch in diesem Fall konnten die einzelnen Lesarten erst anhand der kontextuellen Einbettung der festen Wortverbindung erschlossen werden. Die Resultate der insgesamt 35 Belege zeigen, dass die zweite Lesart zu 69\% dominiert, wie unten im Graph 3 ersichtlich ist.

\section{in Deckung bringen (DWDS-Korpus)}

— 'sich verstecken', 'Schutz gegen Sicht oder Beschuss suchen' 'sich decken, gleich sein ...'

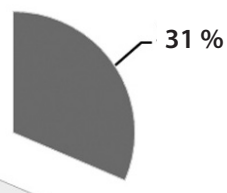

$69 \%$

Graph 3: Der Anteil der Lesarten von in Deckung bringen im DWDS-Korpus 


\section{(c) in Deckung bleiben}

LESART 1: 'sich verstecken', 'Schutz gegen Sicht oder Beschuss suchen' (DUDEN 11)

Beleg 12 Das Ansehen der Kanzlerin lebe im Moment davon, dass sie sich unangreifbar mache, indem sie lange in Deckung bleibt.

(Die Zeit, 12.03.2013)

Beleg 13 Solange das Spiel dauert, ist man sein eigener Herr, entscheidet ständig, welche Laufbahnen man einschlagen will, buchstäblich und metaphorisch: nach vorne stürmen oder in Deckung bleiben, den Weg über links oder rechts oder durch die Mitte wählen.

(Die Zeit, 8.6.2000)

LESART 2: ,sich vor etwas drücken', 'untätig sein“

Beleg 14 Hintergrund ist die Unsicherheit über den weiteren Kurs der US-Notenbank Fed: Solange nicht klar ist, wie lange sie sich noch als großer Bondkäufer auf dem Markt betätigt, bleiben auch andere Investoren lieber in Deckung.

(Die Zeit, 29.10.2013)

Beleg 15 »Mich beunruhigt, dass die Unionsparteien in Deckung bleiben«, sagte Westerwelle.

(Berliner Zeitung, 6.5.2005)

Vor allem in der Finanzwelt und in der Politik drückt die Wortverbindung in Deckung bleiben aus, dass jemand im Moment untätig ist und auf bessere Zeiten bzw. eine erfolgversprechende Angelegenheit wartet. Aufgrund der Ergebnisse wird diese Motivation seltener verwendet und zwar in nur 31\% der insgesamt 35 Belege.

in Deckung bleiben (DWDS-Korpus)

- 'sich verstecken', 'Schutz gegen Sicht oder Beschuss suchen'

'sich vor etwas drücken', 'untätig sein'

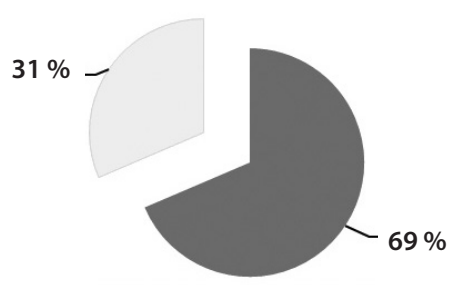

Graph 4: Der Anteil der Lesarten von in Deckung bleiben im DWDS-Korpus 
in Deckung liegen, sein (ziehen, halten)

LESART: 'sich verstecken', 'Schutz gegen Sicht oder Beschuss suchen' (DUDEN 11)

Beleg 16 In der Simulation werden zwei gegnerische Ziele von einem NatoSchiff, das zwischen den Hafenanlagen in Deckung liegt, bekämpft.

(Der Tagesspiegel, 1.3.2002)

Beleg 17 Doch eine der Raketen, vielleicht beide, explodierten, als die Soldaten noch nicht in Deckung waren.

(Der Tagesspiegel, 6.3.2002)

Die weiteren Verben als Komponenten in der Wortverbindung in Deckung + Verb zeugen von den variablen Besetzungen, die von der Intention des Sprachbenutzers abhängen. Allen Verben gemeinsam ist dabei die Lesart 'sich verstecken' sowie der vorwiegend militärische Kontext. Hier gab es m.E. keine andere semantische Interpretation. Die beiden Verben ziehen und halten kommen jeweils in nur einem Beleg vor, so dass sie auch als Varianten aufgefasst werden können.

$$
[\text { die }] \text { Stellung }+ \text { Verb }
$$

Phraseologische Einheiten mit der Komponente Stellung sind auch heute noch eng mit der ursprünglichen Motivation verbunden. Mit Stellung ist im Militärwesen ein ausgebauter oder befestigter Ort im Gelände (Gräben, Stützpunkte, Sperren usw.) gemeint, den die Truppen im Kampf beziehen, um ein Terrain oder sich selbst gegen Angriffe zu verteidigen. Eine ähnliche semantische Motivation findet man auch bei Lexemen wie Feldstellung, Gefechtsstellung, Verteidigungsstellung, Stellungskampf, Stellungskrieg, usw. In Dobniks Tagebuch wurden Wortverbindungen mit der Komponente Stellung an vier Textstellen dokumentiert: Stellung beziehen (2x), Stellung halten, Stellung räumen.

\section{(a) Stellung beziehen}

LESART 1: 'in Bezug auf etw. einen bestimmten Standpunkt einnehmen' (DUDEN 11) 'seine Meinung sagen' (Neumann)

Beleg 18 „Ich werd' zu gegebener Zeit zu allen wichtigen Fragen der Republik Stellung beziehen", wehrt er Neugierige ab.

(Der Tagesspiegel, 8.3.2004) 
LESART 2: 'Soldaten positionieren sich“ 'Stellung gegen Angriffe des Feindes verteidigen und halten"

Beleg 19 Gegen zwölf Uhr wird der Befehl geändert. Das Baon hat eine Stellung zwischen Podsaki und Wojtowszczyzna zu beziehen.

(Dobniks Tagebuch, 2.9.1914)

Wenn man seine Meinung äußert und einen bestimmten Standpunkt einnimmt, dann 'legt man sich fest' bzw. man bezieht eine Stellung. Zu diesem Komponentenbestand könnte auch noch die Präposition $z u$ hinzugefügt werden, weil der Vorfall oder das Problem auch explizit ausgedrückt wird, wie im Beleg 18 bestätigt wird. Dagegen ist im literalen Sinne und in der ursprünglichen Motivation die konkrete räumliche Dimension gemeint, wie es im Beleg 19 aus dem I. Weltkrieg gezeigt wird.

\section{(b) Stellung nehmen}

LESART 1: 'in Bezug auf etw. einen bestimmten Standpunkt einnehmen" (DUDEN 11)

Beleg 20 Bisher hat der Präsident selbst noch nicht Stellung genommen zu den Protesten der Regimegegner, die zum Teil mit brutaler Gewalt beendet worden waren.

(Die Zeit, 28.3.2011)

Beleg 21 Der Papst hat deutlich Stellung genommen gegen jede Leugnung des Holocaust.

(Die Zeit, 5.2.2009)

Stellung nehmen kommt im Tagebuch-Korpus nicht vor. Im DWDS-Korpus wird diese Verbindung nur im phraseologischen Sinne gefunden und verhält sich in der Semantik synonymisch zu der Verbindung Stellung beziehen. Auch in den obigen Belegen werden (fast obligatorisch) die Präpositionen zu/gegen verwendet.

\section{(c) Stellung räumen}

LESART 1: 'seine Position verlassen' (Dobnik)

Beleg 22 Die Russen werden selbstverständlich nicht so dumm sein und uns an der Front angreifen. Diese Kolonne braucht nur noch eine Stunde vorzugehen und wir müssen unsere Stellung ohne Schuß räumen.

(Dobniks Tagebuch, 2.9.1914) 
Beleg 23 Im Gegenzug soll die albanische Untergrundarmee ihre Stellungen räumen.

Wenn ein Ort bzw. eine Stelle zuerst als sicherer Verteidigungspunkt dient und später verlassen wird, um im weiteren Gebiet neue Stellungen zu beziehen, dann wird diese Stellung verlassen bzw. geräumt, wie aus dem Eintrag von Dobnik hervorgeht. Diese Komponente wurde nicht lexikalisiert und ist auch nicht zum phraseologischen Bestandteil der Komponenten um den gängigen militärischen Begriff der ,Stellung` geworden.

\section{(d) die Stellung halten}

LESART 1: '[als Letzter] dableiben und auf etw. aufpassen' (DUDEN 11) 'ausharren' (Neumann)

Beleg 24 Die letzten Offiziere, die noch die Stellung halten, eingepfercht zwischen Isil und Regime, sind verängstigt und verbittert.

(Die Zeit, 9.1.2014)

Beleg 25 Zurück bleiben Großeltern, die sich um ihre Enkelkinder kümmern und auf dem Hof die Stellung halten, bis der Sohn oder die Tochter, so hoffen sie, eines Tages zurückkehrt.

(Die Zeit, 24.1.2013)

Im Zusammenhang mit der Paraphrase 'dableiben und auf etwas aufpassen' wird Stellung halten sowohl im Militärischen als auch im Alltag gebraucht. Der fakultative Gebrauch der Komponenten ,als Letzter' wird in den Belegen 23 und 24 bestätigt, wie aus den einzelnen Lexemen ,die letzten Offiziere' und ,zurückbleiben' hervorgeht. Das Sprachbild der Positionierung in einer geschützten Lage fand Eingang in die Alltagssprache und wird auch weiterhin im übertragenen Sinne verwendet.

\section{(e) etw. in Stellung bringen}

LESART 1: 'etw. für den Einsatz [kampf-, feuerbereit] aufstellen' (DUDEN 11) 'sich (oder etwas) positionieren' (Neumann)

Beleg 26 Gleichzeitig werden Laptop, Telefon und Handy in Stellung gebracht sowie Skype gestartet, ein Internettelefonie-Programm.

(Die Zeit, 11.1.2007) 
Beleg 27 Die Bühnen sind aufgebaut, die Fernsehteams haben sich in Stellung gebracht, die britische Küstenwache wurde in höchste Alarmbereitschaft versetzt.

(Berliner Zeitung, 2.7.2005)

Neumann (2016: 45) wählt für die Beschreibung des Bildes, wenn etwas konkret in eine Position gebracht wird, die Aufstellung der Schneekanonen, die an den Skipisten in Stellung gebracht werden. In den beiden Belegen 26 und 27 sind Sachen und Personen bereit, eingesetzt zu werden, was sich auf zahlreiche Bereiche des Lebens beziehen kann.

\section{6}

\section{ZUSAMMENFASSUNG}

Phraseme sind verfestigte Wortverbindungen, die Sprachbilder in sich tragen. Diese Sprachbilder können beim Sprachbenutzer Assoziationen wecken, Gefühle hervorrufen oder sich auf den Verlauf der Kommunikation auswirken. Wenn wir Phraseme in unserer Kommunikation verwenden, dann fragen wir uns gewöhnlich nicht nach ihrem Ursprung. Gerade dieser Frage sind wir in der vorliegenden Untersuchung nachgegangen, wobei wir uns auf die Suche nach der Motivationsbasis begaben. Wir gingen der Historizität einiger Wortverbindungen nach, die im Soldatenalltag zur Benennung oder Bezeichnung bestimmter Sachverhalte oder Tätigkeiten verwendet wurden. Wir wählten Wortverbindungen mit den Komponenten Feuertaufe, Deckung und Stellung aus dem Kriegstagebuch von Franz Dobnik aus, um sie einem Vergleich zwischen ihrer Verwendung im authentischen Text aus der Zeit des I. Weltkrieges und ihrer Verwendung in der heutigen Alltagssprache bzw. ihren aktuellen Lesarten zu unterziehen. Der Vergleich brachte interessante Erkenntnisse mit sich. Die diachrone Beschreibung von Phrasemen offenbarte diachrone Wandelprozesse, in denen Phraseme auf der semantischen und lexikalischen Ebene bestimmte Veränderungen erfahren hatten. Ein exemplarisches Beispiel veranschaulichen die geschilderten Phraseme mit der Komponente Feuertaufe. Im lexikalischen Bereich zeigte sich erstaunliche Varianz und aus der heutigen Sicht kann die Gebräuchlichkeit der Varianten bestehen, empfangen, erhalten bestätigt werden, während die ursprüngliche Semantik der beiden Lexeme Taufe und Feuer, die auf die Bibelstelle Matth. 3,11 zurückgeht, in Vergessenheit geriet. Heute sind die semantischen Basen, die nur aus einem historischen Textkorpus erschlossen werden können, kaum bekannt. Will man der Historizität der festen Wortverbindungen nachgehen, spielen Realbelege eine wichtige Rolle, weil mögliche Varianten, semantische Motivationsbasis und Gebräuchlichkeit (oft) nur in authentischen Texten zu finden und in historischen Korpora zu überprüfen sind. 


\section{LITERATURVERZEICHNIS}

DUSINI, Arno (2005) Die Möglichkeiten einer Gattung. München: Wilhelm Fink Verlag. DUSINI, Arno (2006) Tagebuch. In: Literaturwisssenschaftliches Lexikon. Grundbegriffe der Germanistik (hrsg. Von Brunner, Horst/ Moritz, Rainer). Berlin: Erich Schmidt Verlag. S. 329.

BRUNNER, Horst/ Moritz, Rainer (2006) Literaturwissenschaftiches Lexikon: Grundbegriffe der Germanistik. Berlin: Erich Schmidt Verlag

BURGER, Harald/Annelies BUHOFER/Ambros SIALM (1982) Handbuch der Phraseologie. Berlin: de Gruyter.

BURGER, Harald (1998) Phraseologie. Eine Einführung am Beispiel des Deutschen. Berlin: Erich Schmidt Verlag.

DOBNIK, Franz (1914) Tagebuch im Fedzug 1914. Privater Besitz.

DUDEN 11 (2007) Redewendungen. Wörterbuch der deutschen Idiomatik. Mannheim: Dudenverlag.

DUDEN online Wörterbuch (http://www.duden.de)

DUDEN (1999) Das große Wörterbuch der deutschen Sprache in 10 Bänden, Mannheim: Dudenverlag.

HARDTWIG, Wolfgang (2005) Der Literat als Chronist. Tagebücher aus dem Krieg 19391945. In: Geschichte für Leser. Populäre Geschichtsschreibung in Deutschland im 20. Jahrhundert (hrgs. von Hardtwig, Wolfgang/Schütz, Erhard). Stuttgart: Franz Steiner Verlag. 147-180.

KLUGE, Friedrich (1883/198922) Etymologisches Wörterbuch der deutschen Sprache. Berlin. New York: de Gruyter.

NEUMANN, Dieter H. (2016) Aufs Korn genommen. Redewendungen aus der Welt des Militärs. Darmstadt: Theiss.

PIIRAINEN, Elisabeth (2012) Widespread idioms in Europe and beyond: toward a lexicon of common figurative units. New York: Peter Lang.

RÖHRICH, Lutz (1994) Lexikon der sprichwörtlichen Redensarten. 5. Bände. Freiburg, Basel, Wien: Herder.

STERGAR, Rok (1999) »Vojski prijazen in zaželen garnizon«: ljubljanski častniki med prelomom stoletja in prvo svetovno vojno. Ljubljana: Zveza zgodovinskih društev Slovenije.

STÖCKL, Hartmut (2004) Die Sprache im Bild - Das Bild in der Sprache. Zur Verknüpfung von Sprache und Bild im massenmedialen Text. Konzepte. Theorien. Analysemethoden. Berlin: de Gruyter.

WISTHALER, Sigrid (2011) Karl Außerhofer - Das Kriegstagebuch eines Soldaten im Ersten Weltkrieg.Innsbruck: innsbruck university press.

WUTHENOW, Ralph-Rainer (1990) Europäische Tagebücher. Eigenart - Formen Entwicklung. Darmstadt: Wissenschaftliche Buchgesellschaft. 


\section{Internetquellen}

CID - christliche internet dienst $\mathrm{GmbH}$

(www.bibel-online.net; Datum des Zugriffs:

23. Juni 2016)

Das Digitale Wörterbuch der deutschen Sprache des 20. Jahrhunderts (DWDS)

(www.dwds.de;

Datum des Zugriffs: 16. 06. 2016)

Digitale Bibliothek der Universität Halle

(http://digitale.bibliothek.uni-halle.de/id/1656695;

Datum des Zugriffs: 8. September 2016)

GRIMM, Jacob und Wilhelm, 1854-1961. Deutsches Wörterbuch von Jacob und Wilhelm

Grimm. 16 Bde. in 32 Teilbänden. Leipzig 1854-1961. Quellenverzeichnis Leipzig 1971.

(http://woerterbuchnetz.de/DWB;

Datum des Zugriffs: 24. August 2016)

Österreichisches Staatsarchiv

(www.archivinformationssystem.at/detail.aspx?ID=88128;

Datum des Zugriffs: 15. September 2016)

POVZETEK

Kaj imajo skupnega Feuertaufe, Deckung in Stellung? Frazemom iz vojaškega vsakdanjika na sledi

Avtorica v prispevku predstavlja stalne besedne zveze s sestavinami Feuertaufe, Deckung in Stellung in njihove spremembe $\mathrm{v}$ leksikalnem in semantičnem razvoju $\mathrm{v}$ diahronem pogledu. Študija se opira na gradivo iz vojaškega dnevnika iz 1. svetovne vojne in zglede iz leksikografskega korpusa DWDS sodobnga nemškega jezika.

Ključne besede: frazeološke sestavine, motivacija, sodobni nemški jezik, vojaški dnevnik, 1. svetovna vojna

\section{ABSTRACT}

What do Feuertaufe, Deckung and Stellung have in common? Some phraseological units from the soldiers' everyday on the track

The article discusses German phraseological units with the components Feuertaufe, Deckung and Stellung and their lexical and semantic changes from the diachronic aspect. The study is based on 
two databases: a German war diary from World War I and the lexicographical corpus DWDS of the contemporary German language.

Key words: phraseological units, motivation, contemporary German, war diary, World War I

\section{ZUSAMMENFASSUNG}

Im vorliegenden Beitrag werden feste Wortverbindungen mit den Komponeten Feuertaufe, Deckung und Stellung unter lexikalischem und semantischem Aspekt dargestellt und einem diachronen Vergleich unterzogen. Als Untersuchungsmaterial dienen das authentische Kriegstagebuch aus dem I. Weltkrieg und Belege aus dem lexikographischen Korpus DWDS der gegenwärtigen deutschen Sprache.

Schlüsselwörter: Phraseologische Komponenten, Motivation, Gegenwartsdeutsch, Kriegstagebuch, I. Weltkrieg 